\title{
Use of thoracic computed tomography by general practitioners
}

\author{
Graham Simpson and Garry S Hartrick
}

S ince its introduction in the 1970s, computed tomography (CT) has revolutionised medical imaging. However, CT scanning involves relatively high exposure to ionising radiation. In the United Kingdom in 1989, CT scans accounted for $2 \%$ of all radiological examinations but contributed $20 \%$ of the total radiation dose to the population from medical use of ionising radiation. By 1998, these figures had risen to $4 \%$ of examinations and $40 \%$ of total dose. ${ }^{1}$ Access to CT scanning is restricted in the UK, whereas in Australia, there is open access for specialists and general practitioners alike. It has been estimated that CT examinations account for $65 \%$ of medical radiation exposure in Australia. ${ }^{2}$

We have observed that many patients referred for a specialist opinion have already had CT scans of the chest that, in many cases, seemed unnecessary. But we did not know in how many cases GPs obtained useful information from a CT investigation that might obviate the need for referral.

\section{METHODS}

Two private radiology practices prospectively recorded requests for CT examination of the chest emanating from the 110 GPs in Cairns between August 2004 and March 2005. Fifty consecutive cases were reviewed by us, after exclusion of requests for CT pulmonary angiography and cases in which the request proved to have originated from a treating specialist. Results of previous plain chest x-rays were obtained and the clinical details reviewed.

We clarified any clinical indications and outcomes, if necessary, by telephone consultation with the requesting GP. We made a subjective assessment as to whether the test was the correct one and whether it was appropriate.

Our study was approved by the Cairns Base Hospital Human Research Ethics Committee.

\section{RESULTS}

The 50 requests came from 36 different GPs, with none requesting more than four $\mathrm{CT}$ scans. Thirty-one of the 50 patients were male; the mean age was 52 years (range, 15-

\section{ABSTRACT}

Objective: To audit requests for computed tomography (CT) examination of the chest emanating from general practitioners and assess the appropriateness and usefulness of these requests.

Methods: We reviewed 50 consecutive requests for CT examination received by two private radiology practices in Cairns between August 2004 and March 2005. Clinical details were abstracted from request forms and clarified by telephone if necessary. A subjective assessment of the appropriateness of the investigation was made by the authors. The study was performed in a large regional centre.

Main outcome measures: Indications for requesting a CT scan; appropriateness of CT scan for indication specified.

Results: Fifteen patients had had recent normal chest x-rays, all of whom proved to have normal CTs; eight had not had a recent chest x-ray performed. The CT scan was considered appropriate in 16 cases (32\%), but 10 of these patients required referral to specialists anyway. Thirty-four CT scans (68\%) were felt to be inappropriate and, of these, 10 were subsequently referred to specialists. In only six cases did the CT scan resolve the GP's clinical problem. In six cases the wrong type of CT scan was performed (five were conventional CT scans instead of high-resolution scans; one was a high-resolution instead of low-resolution scan).

Conclusions: Many CT examinations of the chest requested by GPs could be avoided or replaced by simpler, cheaper tests with lower radiation exposure. Assuming a fatal cancer risk of 1 in 3000, the radiation exposure involved in unnecessary chest CT scans could be responsible for about 40 fatal cancers a year in Australia.

MJA 2007; 187: 43-46

For editorial comment, see page 5

81 years); and six patients were under the age of 35 .

Twenty-seven patients had had an abnormal chest $\mathrm{x}$-ray, 15 had had a recent chest $\mathrm{x}$-ray reported as normal, and eight had not had a recent chest x-ray. The indications for requesting a CT scan are shown in Box 1. All 15 patients with normal chest $\mathrm{x}$-rays subsequently had normal CT scans. Thoracic CT imaging was thought to be appropriate in 16 patients $(32 \%)$. In six of these, no further investigation or referral was needed. The indications and CT findings for these six patients were: post-pneumonic chest x-ray shadowing (shown to be fibrous scarring), suspected bilateral hilar lymphadenopathy (not confirmed on CT), suspected mediastinal widening (not confirmed on CT), a suspected mass on plain chest x-ray (CT normal), pleural thickening (fatty deposits in the pleura), and a small nodule (calcified granuloma on CT).

Twenty patients were subsequently referred for specialist opinion. The CT scan was considered appropriate for 10 of these patients and unnecessary for the other 10 . Four patients were already under specialist care, three with known malignancy and one with common variable immunodeficiency.

Details of the indications for the 34 CT requests thought to be inappropriate are outlined in Box 2. In six cases, the wrong type of CT scan was performed. In five of these, patients with a history of minor asbestos exposure and complaints of breathlessness had conventional CT examinations that would not have demonstrated the interstitial lung disease that was presumably suspected and for which a high-resolution CT (HRCT) would be needed. In the sixth case, an HRCT scan was performed on a patient who had persistent chest x-ray abnormalities after being treated for pneumonia. In this case, HRCT failed to demonstrate the underlying carcinoma that was diagnosed at bronchoscopy some months later (an HRCT scan samples only $10 \%$ of the lung volume and may miss small mass lesions). Some examples of inappropriate CT requests are given in Box 3 . 


\section{Indications for computed tomography scans requested by general practitioners $(n=50)$}

Patients with recent abnormal chest $\mathrm{x}$-ray $(n=27)$

Mass 11

Effusion

Pneumonia

Minor fibrosis

Pleural plaques (asbestos-related)

Atelectasis

Aortic unfolding

Bilateral hilar lymphadenopathy

Bronchial thickening

Suspected mediastinal widening

Interstitial shadowing

Patients with recent normal chest $x$-ray

$(n=15)$

Dyspnoea

Cough

Recurrent infection

Asbestos exposure

Thoracic inlet pain

Ascites

Rectal mucus

Voice change

Patients with no recent chest x-ray $(n=8)$

Known malignancy

Asbestos exposure

Dyspnoea

Haemoptysis

Dysphagia

\section{DISCUSSION}

The weakness of our study is that it depended entirely on subjective and posthoc assessment of the clinical usefulness of an investigation to the requesting doctor by other clinicians. It could be argued, for example, that in all 30 cases in which the patient was not referred to a specialist, the thoracic CT scan provided enough information or reassurance to the GP, and possibly also to the patient, to obviate the need for referral and that the problem was thus solved. Investigators other than ourselves, perhaps including radiologists, may have come to different conclusions about the appropriateness or otherwise of requesting CT scans in individual cases. However, it does seem clear that, in a sizeable proportion of the 50 cases, the thoracic CT scan could have been avoided or replaced with a simpler and cheaper test.

\title{
2 Indications for computed tomography (CT) scans requested by general practitioners for cases in which a CT scan was considered inappropriate or the wrong type of scan was ordered $(n=34)$
}

\author{
Clinical problem as indicated on No. of \\ request form \\ patients Comment
}

I: No imaging indicated

A: No relevant clinical problem to be answered

Dyspnoea (COPD [2], asthma [1], $\quad 54$ CXRs normal (including Case 3, Box 3); 1 CXR smoker [2], unspecified [1]) not done; CT most unlikely to identify cause of breathlessness

Cough

3 All had normal CXRs and trivial symptoms. Unclear what diagnosis could be made by CT

Post-pneumonic effusion

1 Appropriate investigation was aspiration of fluid. Having CT delayed drainage of empyema

Suspected aortic unfolding

1 Common normal variant, not a disease

Thoracic inlet pain

1 Unclear what diagnosis could be made on CT

Recurrent infection

1 Bronchoscopy indicated if obstructing lesion suspected

Ascites

Rectal mucus

17

Dysphagia

1 Non-respiratory condition; not clear what chest $\int \mathrm{CT}$ would contribute

B: No change in diagnosis or management likely

$\left.\begin{array}{ll}\text { Known cancer } & 3 \\ \text { Common variable immunodeficiency } & 1\end{array}\right\} \begin{aligned} & \begin{array}{l}\text { Patients already under specialist care. One case } \\ \text { terminal (Case 5, Box 3) }\end{array} \\ & \text { Lung cancer } \\ & 2\end{aligned} \quad \begin{aligned} & \text { Both cancers clinically inoperable (Pancoast } \\ & \text { tumour [1], vocal cord palsy [1]) }\end{aligned}$

II: Incorrect choice of technique

Dyspnoea, with history of asbestos exposure

5 Conventional CT performed in all cases, but HRCT needed to demonstrate interstitial lung disease

Pneumonia follow-up

2 CXR sufficient (Case 1, Box 3). One patient had HRCT, which failed to show underlying lung cancer (HRCT samples only $10 \%$ of lung volume)

Minor fibrosis/atelectasis

2 Repeat CXR was, or would have been, adequate

Haemoptysis; contact with TBpositive person

$1 \quad \mathrm{CXR}$ and sputum examination needed instead (Case 2, Box 3)

Bronchial thickening

1 Repeat CXR suggested by radiologist but ignored

III: Correct choice of imaging but wrong timing

Nodule seen on CXR 1 Patient had four CTs in 4 months and was then discharged. Repeat CXR or CT in 6 months would have been appropriate (Case 4, Box 3)

$\mathrm{COPD}=$ chronic obstructive pulmonary disease. $\mathrm{CXR}=$ chest $\mathrm{x}$-ray. $\mathrm{HRCT}=$ high-resolution computed tomography. $\mathrm{TB}=$ tuberculosis.

It was clear from discussions with the GPs involved that most had no training in or knowledge of the indications for CT scanning or the uses of conventional as opposed to high-resolution scans. In many cases it was not clear what diagnosis was being sought. A plain chest x-ray is actually a very sensitive investigation, except in some rare conditions (such as diffuse interstitial lung disease), and it is noteworthy that, in all 15 cases in which a preceding chest $\mathrm{x}$-ray was reported as normal, the subsequent CT scan simply confirmed normality. There seems to be no excuse for going straight to thoracic CT scanning without performing a chest $\mathrm{x}$ ray first. In some cases it was clear that the GP requested the CT scan even though he or she had already decided on specialist refer- 
ral. This was presumably done in order to save the specialist time. However, even in these cases, half of the CTs were felt to be inappropriate. The fact that in only six of 50 cases the CT scan seemed to be appropriate and to have solved the GP's clinical problem is alarming.

In 2004, there were 110 full-time equivalent GPs in Cairns for a population of around 100000 , giving a ratio of 909 patients to one GP. This compares with a national average of 1223 patients per GP, assuming the Australian population to have been 20 million in $2004 .^{3}$ There were three respiratory physicians in practice at the time of our study, with waiting times for routine referrals of 2-3 weeks (urgent cases would usually be seen in 1-2 days). Thus, neither excessive workload for GPs nor poor access to specialist opinion explains the pattern of referral for CT scans.

There is no reason to believe that GPs in Cairns are different in ability or training from those elsewhere in Australia. We do not have enough data to assess the impact of various levels of GPs' vocational training on referral patterns. Discussions with GP colleagues suggest that pressure from patients and a fear of litigation for missed diagnoses are important factors influencing imaging requests.

There have been no comparative studies looking at GP versus specialist practice in this area. However, in a similar study at Cairns Base Hospital, only 29 of 50 requests (58\%) for chest CTs on hospital inpatients by non-respiratory specialists and junior doctors were found to be appropriate, suggesting hospital practices also need review. ${ }^{4}$ It has previously been reported that doctors in general have a very poor knowledge of the doses of ionising radiation involved in a range of radiological investigations. ${ }^{5}$

Newer multislice helical scanners offer potential for dose-reduction techniques. It is difficult to assess how much impact these techniques will have on total radiation exposure, particularly as helical scanning with later reconstruction of thin slice images is now often used instead of the lower-dose traditional HRCT.

Ideally, all requests for high-dose radiological examinations should be reviewed by a radiologist before the test is performed. This may be difficult, particularly for private radiologists, who could also be perceived as having some conflict of interest. Nevertheless, increasing or formalising radiologists' involvement in vetting requests may be a means of improving standards.

\section{Examples of inappropriate requests for a computed tomography (CT) scan}

Case 1: A 15-year-old girl had a mild community-acquired pneumonia. The radiologist suggested a repeat chest $x$-ray (CXR) to confirm clearing, but a CT scan was performed instead. A plain CXR would have been perfectly adequate and would have exposed the patient to a much lower radiation dose.

Case 2: A 62-year-old man whose wife had had strongly smear-positive pulmonary tuberculosis (TB) 1 year earlier presented with haemoptysis. Initial contact screening had been inconclusive. No CXR was performed, but a CT scan was reported as showing probable carcinoma. When the patient was subsequently referred to the hospital chest clinic for bronchoscopy, a CXR showed classical changes of pulmonary TB, which responded clinically and radiologically to standard TB treatment. There was no evidence of malignancy.

Case 3: A 25-year-old non-smoking woman with severe asthma from childhood presented complaining of breathlessness and wheeze and had a normal CXR. A later CT scan to further investigate her dyspnoea was also normal and did not contribute to the diagnosis of an exacerbation of asthma.

Case 4: A 40-year-old man had a CXR because of a cough. An incidental finding was a small upper lobe nodule that was confirmed on CT scan. The general practitioner organised four CT scans to follow the progress of the nodule over the next 4 months, then discharged the patient from further follow-up, as the nodule had not changed.

Case 5: A 76-year-old man with terminal metastatic lung cancer was under the care of the palliative care team. He had already had radiotherapy, chemotherapy, talc pleurodesis and numerous alternative therapies, and was now dying. The CT scan, requested to assess the progress of the tumour, was inappropriate, as the result would not have altered management.

Medicare Australia statistics show that over 235000 CT scans of the chest, including scans also involving other areas such as the abdomen, were performed by private radiology practices in the financial year 2004-05. This excludes all CT scans performed in public hospitals, those billed to the Department of Veterans' Affairs, and those involving requests for CT pulmonary angiography. The cost to the taxpayer in Medicare rebates for these examinations was over $\$ 82$ million. It is not known exactly how many of these requests emanate from general practice. In Cairns there are two private radiology suppliers. One practice is attached to Cairns Private Hospital and estimates that $50 \%$ of its requests for chest CT scans come directly from GPs. The other practice estimates that up to $90 \%$ of requests for chest CT scans come from general practice. Assuming that 70\% of requests (the average of the estimates from the two radiology practices) come from GPs and that two-thirds are inappropriate, this means that there may be an annual cost to Australian taxpayers of over $\$ 35$ million for unnecessary CT examinations of the chest.

The risks of overexposure to medical use of ionising radiation are real. A chest x-ray gives an exposure of about $0.02 \mathrm{mSv}$, whereas a CT scan of the chest typically gives an exposure of around $8 \mathrm{mSv}$, or the equivalent of exposure to 400 plain chest $\mathrm{x}$ rays. ${ }^{1,6-9}$ The dose delivered to breast tissue from a conventional CT chest examination may be as high as $33 \mathrm{mSv}{ }^{10}$ The International Commission on Radiological Protection estimates an overall risk of inducing a fatal cancer as $6.0 \%$ per sievert in the whole population and $4.8 \%$ per sievert in adults. ${ }^{11}$ This equates to a risk of about one cancer per 2000-3000 chest CT scans for doses in the range involved in chest CT scans. This risk is age-related, with exposure earlier in life carrying a greater risk. ${ }^{12}$ For adults, in whom the background incidence of tumour is relatively high, the proportional increase in risk is low. However, even a small increase in relative risk will produce a meaningful increase in malignancies, given the vast numbers of CT examinations now performed. Assuming again that 70\% of requests come from general practice and that two-thirds are unnecessary, and taking an overall cancer risk of 1 in 3000 scans, we estimate that about 40 fatal cancers may be caused annually in Australia by CT examinations of the chest that could have been avoided. We suggest that further audits of the appropriateness and utility of CT examinations in the community should be undertaken, not only of chest CT scans but also of CT scans of other regions such as the abdomen, head and lumbar spine.

\section{ACKNOWLEDGEMENTS}

We thank the staff of North Queensland X-ray Services and Queensland X-Ray for their help and cooperation. 


\section{IMPROVING PRACTICE}

\section{COMPETING INTERESTS}

None identified.

\section{AUTHOR DETAILS}

Graham Simpson, MD, FRACP, FRCP, Director, ${ }^{1}$

Clinical Associate Professor ${ }^{2}$

Garry S Hartrick, MB BS, Chair ${ }^{3}$

1 Thoracic Medicine and Regional TB Control

Unit, Cairns Base Hospital, Cairns, QLD.

2 Department of Medicine, James Cook University, Cairns, QLD.

3 Cairns Division of General Practice, Cairns, QLD.

Correspondence: fgsimpson@iig.com.au

\section{REFERENCES}

1 Rehani M, Berry M. Radiation doses in computed tomography. The increasing doses of radiation need to be controlled. BMJ 2000; 320: 593-594.

2 Wise KN, Thomson JEM. Changes in CT radiation doses in Australia from 1994 to 2002. The Radiographer 2004; 51: 81-85.

3 Health Workforce Queensland. Overview of the Queensland general practice workforce 20042005. Brisbane: HWQ, 2005

4 Gunes A, Simpson G. Utility of CT chest scanning in the inpatient setting [abstract]. Respirology 2006; 11 (Suppl 2): A69.

5 Shiralkar S, Rennie A, Snow M, et al. Doctors' knowledge of radiation exposure: questionnaire study. BMJ 2003; 327: 371-372.

6 Shrimpton, PC, Jones DG, Hillier MC, et al. Survey of CT practice in the UK. Part 2: Dosimetric aspects. London: HMSO, 1991.

7 Allan PL, Williams JR. Full-body CT scans: are they worth the costs and radiation exposure? J R Coll Physicians Edinb 2003; 33: 8-14.
8 Royal College of Radiologists. Making the best use of departments of clinical radiology: guidelines for doctors. 4th ed. London: RCR, 1998.

9 Committee 3 of the International Commission on Radiological Protection (ICRP). Radiation and your patient: a guide for medical practitioners. http://www.icrp.org/docs/Rad_for_GP_ for_web.pdf (accessed Mar 2007).

$10 \mathrm{McC}$ collough $\mathrm{CH}$, Liu HH. Breast dose during electron-beam CT: measurement with film dosimetry. Radiology 1995; 196: 153-157.

11 International Commission on Radiological Protection. 1990 Recommendations of the International Commission on Radiological Protection. Oxford: Pergamon, 1991.

12 Brenner DJ, Elliston CD, Hall EJ, Berdon WE. Estimated risks of radiation-induced fatal cancer from pediatric CT. AJR Am J Roentgenol 2001; 176: 289-296.

(Received 7 Mar 2006, accepted 24 Jan 2007) 